\title{
Lateral Transtumoral Transforaminal Removal of Cervical Dumbbell Tumors
}

\author{
Sudhir Dubey ${ }^{1}$ Amit Agrawal ${ }^{2}$ \\ ${ }^{1}$ Division of Minimally Invasive Neurosurgery, Medanta Institute of \\ Neuroscience, Medanta The Medicity, Sector 38, Gurgaon 122001, \\ Haryana, India \\ 2Department of Neurosurgery, Narayna Medical College Hospital, \\ Chinthareddypalem, Nellore 524003, Andhra Pradesh, India \\ Indian J Neurosurg 2022;11:179-184
}

\begin{abstract}
Address for correspondence Dr. Sudhir Dubey, MCh, Division of Minimally Invasive Neurosurgery, Medanta Institute of Neuroscience, Medanta the Medicity, Sector 38, Gurgaon 122001, Haryana, India (e-mail: sudhir6321@yahoomail.com).
\end{abstract}

\begin{abstract}
Background Cervical dumbbell tumors constitute $15 \%$ of cervical nerve sheath tumors. These tumors occupy intraspinal, foraminal, and extraspinal compartments. Laminectomy, laminectomy and facetectomy and anterolateral partial vertebrectomy are the approaches used to address these multicompartment tumors. Laminectomy leads to incomplete tumor removal. Other approaches ensure complete tumor excision but involve more extensive bone work.

Objective We describe our surgical technique and clinical experiences of six patients treated through single lateral approach, addressing all compartments (transforaminal approach) with minimal or no bony resection.

Methods Six patients with cervical dumbbell tumors underwent minimally invasive transforaminal approach and tumor excision. The cohort consisted of four women and two men. All patients underwent complete tumor excision

Technique Intraoperative $\mathrm{X}$-ray and ultrasound are used to localize the respective foramen. Skin is entered from the lateral side and sequential dilatation is done through muscles. The dilators and port are docked to the lateral surface of articular facets. Standard technique of capsule dissection, internal decompression and capsule mobilization is done to remove the tumor. Arachnoid is seen at the end. Tumor cavity is obliterated with fat graft.

\section{Keywords}

- cervical spine

- dumbbell tumors

- lateral approach

- neuromas

Results Total excision of tumor was documented in all patients with postoperative MRI scan. The mean blood loss was $100 \mathrm{~mL}$. The patients could be discharged on second or third postoperative day. Mean follow-up is of 48 months (18-70 months) and all patients had resolution in features of myelopathy.

Conclusions Endoportal transforaminal approach is technically feasible and minimally invasive option to remove the dumbbell schwannomas.
\end{abstract}

\section{Introduction}

The tumors of the spinal nerve sheath, namely, neurofibroma, neurilemma, and schwannoma are the most

published online

February 4, 2022
DOI https://doi.org/

$10.1055 / \mathrm{s}-0040-1721875$

ISSN 2277-954X. commonextramedullary tumors of the cervical spine. They usually arise from the dorsal sensory root near the edge of its exit from the spinal canal and thus are intraspinal. However, (c) 2022. Neurological Surgeons' Society of India.

This is an open access article published by Thieme under the terms of the Creative Commons Attribution-NonDerivative-NonCommercial-License, permitting copying and reproduction so long as the original work is given appropriate credit. Contents may not be used for commercial purposes, or adapted, remixed, transformed or built upon. (https://creativecommons.org/licenses/by-nc-nd/4.0/)

Thieme Medical and Scientific Publishers Pvt. Ltd., A-12, 2nd Floor, Sector 2, Noida-201301 UP, India 
$15 \%$ of these follow the nerve sheath through the intervertebral foramen. ${ }^{1}$ Because the narrowest portion of the course of the nerve root is at its exit from the bony spinal canal, tumors that develop both inside and outside the spinal canal have a dumbbell appearance. This is radiographically typical, particularly when the bone around the nerve root foramen is eroded. Some of these will go on to develop large extraspinal components. Management of cervical dumbbell tumor holds a challenge because of unique exposure requirements, proximity of the vertebral artery, potential spinal instability (owing to bony erosion by the tumor and the bony removal during surgery), and the risk of upper extremity nerve root injury. ${ }^{2}$ To deal with these tumors, posterior (laminectomy), posterolateral (laminectomy with facetectomy), ${ }^{2}$ and anterolateral ${ }^{3-6}$ approaches have been advocated. The contiguous intraspinal, foraminal, and extraforaminal components make this multicompartment and involve resecting a part of vertebral body in the form of unilateral facetectomy or extended foraminotomy. Excellent results have been achieved by these approaches. ${ }^{1-6}$ These tumors have been classified according to an anatomical spread by Eden, ${ }^{7}$ Asazuma ${ }^{8}$ and Jiang ${ }^{9}$ (PUTH, Peking University Third Hospital). These classifications help in determining the appropriate surgical approach to enable complete excision of the tumor. The most important challenges in long-term follow-up are recurrences and cervical deformity. ${ }^{9}$ These concerns are best addressed initially in the treatment plan. We describe our surgical technique and clinical experiences of six patients treated through single lateral approach, addressing all compartments (transforaminal approach) with minimal or no bony resection.

\section{Material and Methods}

Six patients with cervical dumbbell tumor underwent minimally invasive transforaminal excision between May 2005 to February 2010. The median age of presentation was 34 years. Four women and two men presented with features of radiculopathy $(n=2)$ and myelopathy $(n=6)$. MRI scan with gadolinium enhancement was performed to assess the size, extent of lesion, and status of vertebral artery. CT scan with bone windows was done to see the integrity of articular facets. Adequate information about the vertebral artery could be obtained from MRI; therefore, no patient underwent digital subtraction angiography (DSA). All patients had intradural and intraforaminal involvement. Tumors on MRI scan were a mixed picture of PUTH Type 1 and Type 3 or Asazuma Type IIA or B. Two patients with extraforaminal spread also had erosion of the facet. Two patients had tumor at C4-5 level, while the rest of the levels addressed were $\mathrm{C} 1-2, \mathrm{C} 2-3$, C3-4 and C56.

Intraoperative direct motor-evoked potential (MEP) monitoring was done on the side of the lesion in all muscles supplied by that root and contiguous roots above and below. Transcortical MEP was measured in both the lower limbs. A $2.5 \mathrm{~cm}$ diameter tube was used to approach the lesions, and depth varied with distance of facet from the skin. Gross total excision could be achieved in all six patients. Two patients had erosion of articular facets of two adjoining vertebrae, and they underwent reverse of lateral mass fusion (screws were placed from ventral to dorsal direction in the lateral mass in contrast to dorsal to ventral direction as in conventional approach) through the same approach to avoid instability. The fat graft was placed in the empty foramen, and the wound was closed in layers. Pathological findings revealed schwannoma ( $n=5)$ and neurofibroma $(n=1)$. The patient with all three compartments and articular facet involvement had preoperative C5 motor weakness and the nerve root could not be preserved. Root could be preserved only in two cases but could not be identified in rest of the cases.

\section{Operative Technique}

After intubation, the patient is positioned in the lateral position. Slight lateral flexion is given to open the intervertebral foramen. C-arm and ultrasound are used to localize the level of intervertebral foramen at the level of pathology. A skin incision of $2.5 \mathrm{~cm}$ is placed on the posterolateral aspect of neck. The trajectory is directed to lateral surface of articular facets. After the investing layer of the deep fascia of the neck is incised, muscles are seen. Through these muscles, a dilator is passed under fluoroscopic guidance and articular facet is felt. Toggling is done to create a plane in superior inferior direction and anterior to articular facets. This is followed by sequential dilatation. An expandable self-retaining tubular $(2.4 \mathrm{~cm}$ diameter) retractor is placed and fixed $(\boldsymbol{-}$ Fig. $\mathbf{1 A})$. The length of retractor can vary, depending on depth of facet from skin.

\section{Extraforaminal and Foraminal Tumor}

The tumor is seen at the distal end of the retractor. The capsule is inspected for the presence of ventral roots. Direct-evoked potential can be elicited on the tumor capsule. The capsule

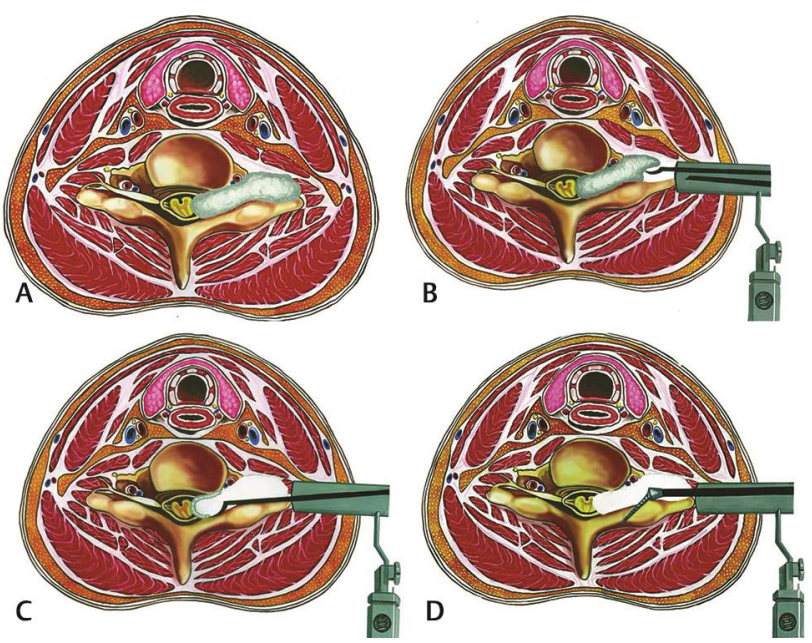

Fig. 1 Transforaminal approach. (A) Shows the cervical dumbbell neurofibroma causing widening of the intervertebral foramen and displacing the vertebral artery and the spinal cord. (B) Shows the trajectory of the port from posterolateral aspect. (C) The tumor is internally debulked and capsule is mobilized until finally a small capsule is left over the arachnoid, which is slowly dissected from the arachnoid. (D) Shows the trajectory of the screw placed if the facet is eroded by the tumor 
is cut and internal decompression done. It is essential to the spatial plane of the structures around the area where the surgery is being performed. Ventrally, the vertebral body with vertebral artery, anterior dural sheath and the ventral roots are present, while dorsally, the articular facets are present. The principles of microsurgery-internal decompression, capsule mobilization and further decompression are followed ( - Fig. 1B).

\section{Removal of the Intraspinal Part}

Once the foramen is reached, tactile inputs from instrument are used as guide to lead to spinal canal. The articular processes dip and the vertebral body forms a knuckle as spinal canal is entered. The tumor is then debulked in center and capsule mobilized with curved dissector around the edges. There is brisk epidural ooze as soon as junction of foramen and spinal canal is reached. As increasing debulking is done, the amount of capsule that can be mobilized increases. Depth for debulking can be assessed by mobilizing the capsule and assessing the medial extent of the tumor. Finally, arachnoid is seen (-Fig. 1C). Medial to arachnoid are nerve roots. An attempt should be made to preserve the arachnoid. Within the arachnoid, the spinal cord, blood vessels and nerve roots are seen. Arachnoid could be preserved in three cases.

\section{Reverse Lateral Mass Fixation}

The decision for fusion is taken on basis of preoperative CT scan. If there is complete erosion of any facet, it is decided to fuse with reverse lateral mass fixation. For this, the normal thick articular processes are exposed above and below ( -Fig. 1D). High-speed drill is used to make initial hole on anterolateral surface of facet. The trajectory is directed toward spinous processes. The direction of the screw should be away from visualized cord or arachnoid. This trajectory requires slight anterior toggling of the port. Fourteen mm screws are passed after drilling is completed and rod is connected. Bone autograft is taken from lateral part of facets.

\section{Closure}

Fat is placed in the foramen and the wound is closed in layers. The closure of deep fascia of neck should be water-tight

\section{Results}

Postoperative contrast MRI was done to document excision. Complete tumor resection was performed in all patients. No patient complained of neck pain at follow-up. Mild operative site pain was noted, which resolved by the time of discharge. One patient with short neck developed neck spasm after 5 days and required 2 weeks of physiotherapy for pain to resolve. There was no cerebrospinal fluid (CSF) leak, and no drainage lumbar puncture was required. Mean follow-up is of 48 months. All patients had resolution of myelopathy features. One patient continued to have persistent weakness in $\mathrm{C} 5$ dominant root and required intercostobrachial anastomosis at follow-up. The details of the patients are summarized in - Table 1 .

\section{Illustrative Cases}

Case 1: A 68-year-old gentleman reported neck pain predominantly on right side for a duration of 1 year. He also had stiffness and difficulty in walking with both lower limbs (right side being worse than left side). His MRI cervical spine with intravenous (IV) gadolinium showed a C34 dumbbell tumor ( $\mathbf{F i g .} \mathbf{2 A}, \mathbf{B}, \mathbf{C}$ ). The tumor had displaced the spinal cord to the opposite side in the intraspinal part, vertebral artery anteriorly and medially in the intraforaminal part and was projecting laterally as it comes out of the foramen. It was decided to address the tumor with the transforaminal approach (Fig). The surgery and the postoperative course were uneventful, and the patient was discharged in stable condition. Repeat contrast MRI did not show any residual tumor ( - Fig. 2D,E,F).

Case 2: A 74-year-old lady presented with features of myelopathy of 3 months duration. Her CT scan showed

Table 1 Clinical characteristic of the patients

\begin{tabular}{|l|l|l|l|l|l|l|l|l|l|}
\hline No & Age/Sex & $\begin{array}{l}\text { Clinical } \\
\text { presentation }\end{array}$ & $\begin{array}{l}\text { Duration } \\
\text { of } \\
\text { symptoms } \\
\text { (months) }\end{array}$ & Level & $\begin{array}{l}\text { Follow-up } \\
\text { duration } \\
\text { (months) }\end{array}$ & $\begin{array}{l}\text { Preoperative } \\
\text { myelopathy } \\
\text { (Nurick's) }\end{array}$ & $\begin{array}{l}\text { Postoperative } \\
\text { myelopathy } \\
\text { (Nurick's) }\end{array}$ & $\begin{array}{l}\text { Deformity } \\
\text { at } \\
\text { follow-up }\end{array}$ & Pathology \\
\hline 1 & $68 \mathrm{Y} / \mathrm{M}$ & $\begin{array}{l}\text { Myelopathy, } \\
\text { neck pain }\end{array}$ & 18 & C3-4 & 70 & 4 & 1 & None & Schwannoma \\
\hline 2 & $74 \mathrm{Y} / \mathrm{F}$ & $\begin{array}{l}\text { Myelopathy, } \\
\text { radiculopathy }\end{array}$ & 13 & C5-6 & 45 & 3 & 1 & none & Schwannoma \\
\hline 3 & $45 \mathrm{Y} / \mathrm{F}$ & $\begin{array}{l}\text { Myelopathy, } \\
\text { radiculopathy }\end{array}$ & 8 & C4-5 & 54 & 2 & 0 & none & Schwannoma \\
\hline 4 & $23 \mathrm{Y} / \mathrm{F}$ & $\begin{array}{l}\text { Myelopathy, } \\
\text { neck pain }\end{array}$ & 6 & $\mathrm{C} 2-3$ & 49 & 2 & 0 & None & Neurofibroma \\
\hline 5 & $20 \mathrm{Y} / \mathrm{M}$ & Myelopathy & 12 & $\mathrm{C} 4-5$ & 52 & 3 & 0 & None & Schwannoma \\
\hline 6 & $18 \mathrm{Y} / \mathrm{F}$ & Myelopathy & 9 & $\mathrm{C} 1-2$ & 18 & 3 & 0 & None & Schwannoma \\
\hline
\end{tabular}



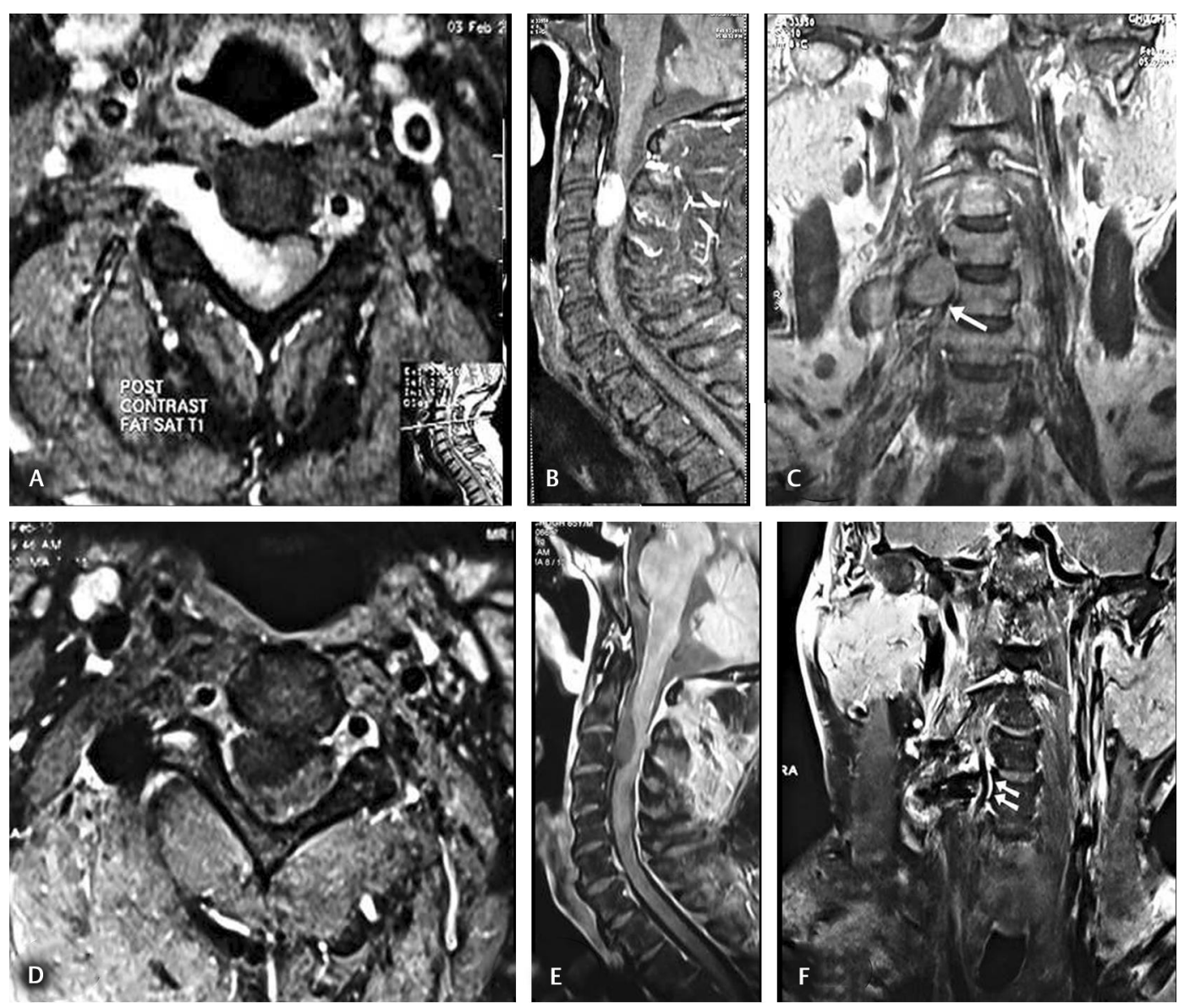

Fig. 2 Patient 1, 69-year-old man presented with progressive quadriparesis. (A) Preop T1-weighted contrast MRI scan (axial) shows the dumbbell tumor. (B) Preop T1-weighted contrast MRI scan (sagittal) shows the dumbbell tumor. (C) Preop T2-weighted coronal MRI scan shows the dumbbell tumor displacing the vertebral artery. (D) Postop T1-weighted contrast MRI scan (axial) shows complete tumor removal. (E) Postop T1-weighted contrast MRI scan (sagittal) shows complete tumor removal. (F) Postop T1-weighted contrast coronal MRI scan shows the dumbbell tumor cavity artifacts with normal vertebral artery.

destruction of the articular facet with widening of neural foramen ( - Fig. 3A). MRI showed dumbbell tumor ( - Fig. 3B,C). The tumor was removed by transforaminal approach ( Fig. 4A-D). Postop X-rays ( - Fig. 3E,F), and MRI ( - Fig. 3D) were done, which showed total tumor removal. Patient had a resolution of symptoms at 1 -year follow-up.

\section{Discussion}

The dumbbell tumors of the spine are multicompartment tumors. They occupy intraspinal, intraforaminal, and extraforaminal compartments. The cervical spine is unique, as it can be approached in all 360 degrees, respecting the neurovascular and visceral structures.

Various approaches have been described to deal with the dumbbell tumors. The commonly used conventional approach used is laminectomy. But the limitation of this approach is that it does not address the intraforaminal component. Subtotal removal of the tumor results in recurrence in 1 to 9 years duration (mean 4.1 years). There may also be rapid growth of tumor remnant. ${ }^{4}$ Some surgeons combine laminectomy with medial facetectomy. The enlarged intervertebral foramina make a natural corridor for removal of foraminal component. This can address only tumors with limited foraminal extension. Posterior subcapsular approach to brachial plexus and its roots has been described..$^{10}$ Although this approach can provide extensive access to the plexus till the foramen, it provides limited access to intraspinal foramen. McCormick has described posterolateral approach to lesions in these locations. ${ }^{11}$ They suggest laminectomy with complete facetectomy or hemilaminectomy with complete facetectomy to address these tumors. There are several advantages with this approach. The tumors generally arising from the dorsal roots, the ventral roots and dural sleeve protect the vertebral artery. The plane can be made from the ventral roots proximally in the spinal canal. The surgeons are familiar with this approach. Lateral mass fusion, although required on one side, is a familiar procedure and does not limit the range of motion significantly.

Anterolateral approaches have been described by several authors. These are modification of the lateral approach to cervical spine for spondylotic disease by Verbiest. ${ }^{6}$ The approach is lateral to carotid artery in the neck. It provides the exposure of transverse process, uncovertebral joint, and lateral 

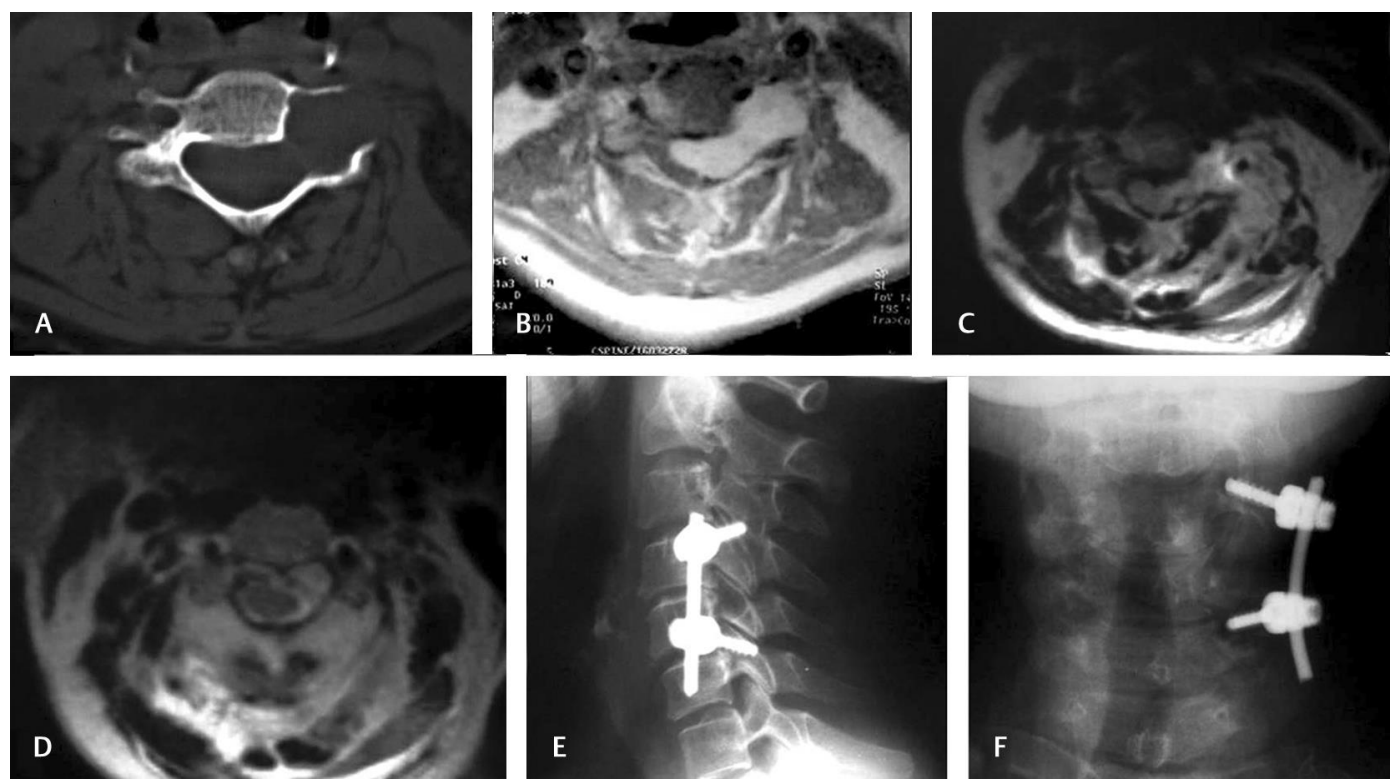

Fig. 3 Patient 2, 74-year-old lady presented with progressive quadriparesis. (A) Preoperative CT scan shows destruction of the facet and widening of the intervertebral foramen. (B,C) Preoperative MRI axial cuts plain and post contrast shows the dumbbell neurofibroma displaying the vertebral artery and spinal cord. (D) Postoperative MRI axial scan shows tumor removal. (E,F) Postoperative X-ray lateral and AP view shows reverse lateral mass fixation.
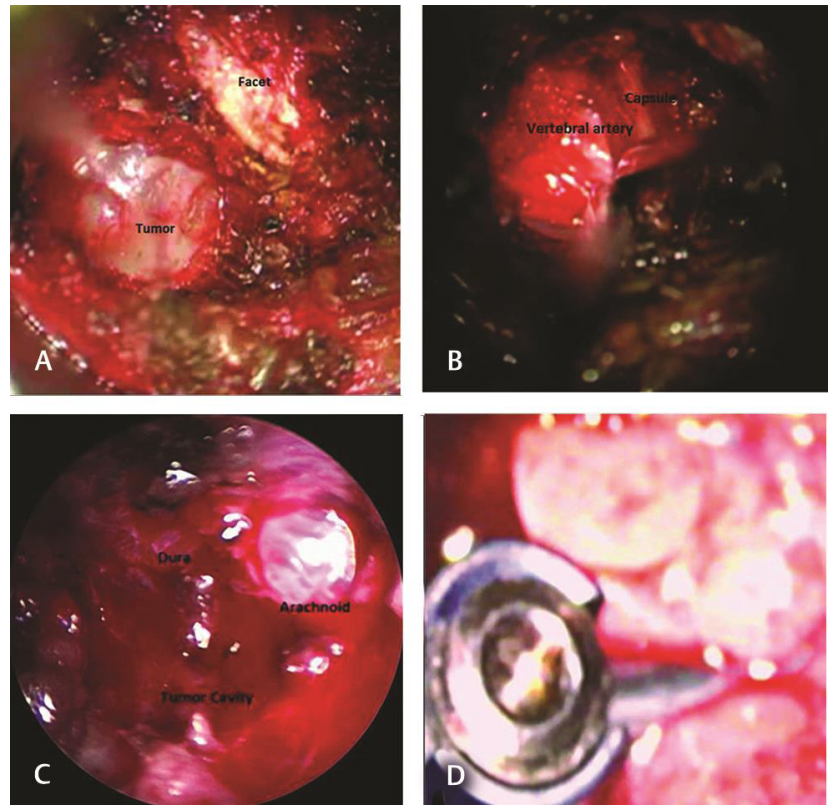

Fig. 4 Intraoperative photograph. (A) Shows the articular facet and tumor capsule through the port. (B) Shows the dissection of the tumor capsule over the vertebral artery. (C). Shows the foraminal anatomy after tumor removal in the depth of tumor cavity arachnoid and dura can be seen. (D) Shows the screws, rod, and the fat graft.

vertebral body. Vertebral artery can be mobilized and controlled by this approach. The dissection of vertebral artery in foramen transversorium can be bloody, due to venous plexus, and time consuming. For intraspinal pathology, oblique corpectomy will be required. Also, injury to phrenic nerve, accessory nerve, and sympathetic plexus may occur with this approach. The advantages of this approach are the extensive access to the brachial plexus and the proximal nerve roots as well as early visualization and control of the vertebral artery.

Lateral transforaminal approach is a minimally invasive procedure that involves muscle splitting with a $3 \mathrm{~cm}$ long skin incision. The muscles are split and not dissected subperiosteally. Once the tumor is reached, the corridor created by tumor is used to resect further tumor. The principles of internal decompression followed by capsule mobilization are kept in mind. The tactile information from articular facets and the vertebral body helps to know when spinal canal is reached. Once decompression has been achieved till the inside of the foramen, the capsule is cut looking for nerves till that level. Further central debulking with the CUSA and lateral capsule mobilization is done. No pressure should be put on the tumor, and it should be delivered out. The nerve roots are seen splayed with arachnoid between tumor and nerve roots

Dumbbell tumors cause progressive erosion of articular facets. ${ }^{8,9}$ Complete loss of facet joint and ligamentous structures puts the patients at risk for spinal stability. This leads to progressive scoliosis or kyphoscoliosis. As much as $50 \%$ of patients who undergo facetectomy develop progressive deformity. From an average preoperative lordosis of 17 degrees, the average postoperative kyphosis is 8 degrees and scoliosis is of 25 degrees. ${ }^{9}$ Only some will require surgery to correct the deformity. ${ }^{8,9}$ Minimally invasive transforaminal approach does not cause additional instability, as bone removal is negligible. It also enables us to correct the instability preemptively if there is facetal erosion or if facet requires to be drilled in case of narrow foramen.

Various anatomical classifications have been developed for dumbbell tumors by Eden, ${ }^{7}$ Asazuma ${ }^{8}$ and Jiang ${ }^{9}$ (PUTH). These have been devised to form surgical strategy, in order to 
address various compartments of the tumor. Minimally invasive transforaminal approach can be used in cases in which the foramen is sufficiently and uniformly widened to deliver intraspinal tumor. In cases where it is not widened enough, further drilling of facet is possible. This can be combined with fusion procedure in the same sitting. Tumors that extend for more than one intervertebral foramen, tumors that are primarily intraspinal (Asazuma Type I, PUTH Type1 and Type2), and tumors with no intraforaminal components (Asazuma Type IV and Type V, PUTH Type 4) are not amenable for transforaminal approach.

The neuromas are the most common extramedullary tumors of the spinal canal. Majority of them lie within the spinal canal. Only $15 \%$ extend laterally within the dural sheath of nerve of origin and have both intradural and extradural component. ${ }^{1}$ Few tumors may arise from proximal extradural spinal nerve in the foraminal or extraforaminal region and grow concentrically and proximally. In these cases, the intraspinal component may be entirely extradural.

More than $80 \%$ of neuromas are solitary sporadically occurring schwannomas; $15 \%$ are neurofibromas. More than two-thirds of neurofibromas are associated with von Recklinghausen's disease. They exhibit dumbbell growth in $66 \%$ of cases. $^{12}$ Plexiform neurofibromas are rare and may extend proximally into the subpial compartment. ${ }^{11}$

With the exception of plexiform neurofibromas, benign nerve sheath tumors arising from extradural spinal nerve, plexus or peripheral nerves are well-encapsulated. They may splay the functioning nerve fascicles on surface or they may be embedded in the capsule. The fascicles running into the tumor are nonfunctional.

In intradural spinal tumors, the fascicles disappear into the substance of tumor as the roots do not have connective tissue matrix. It is only possible to preserve nerve root fascicles in small intradural nerve sheath tumors. In these tumors, the arachnoid layer is present between the motor and sensory root but distal to the dorsal root ganglia; sacrifice of entire motor and sensory root is required as no arachnoid layer is present. ${ }^{9}$

\section{Conclusions}

Transforaminal approach adds an additional dimension in the armamentarium of spinal surgeons. It preserves the normal anatomical structures while achieving complete tumor resection. This prevents spinal instability as no bone work is involved. Complete excision avoids recurrence.

\section{Funding}

None.

\section{Conflict of Interest}

None declared.

\section{References}

1 Nittner K, Spinal meningiomas, neuromas and neurofibromas and hourglass tumors In Pierre HV, George WB, eds Handbook of Clinical Neurology New York, North Holland/America: Elsevier; 1976:177-322

2 McCormick PC. Surgical management of dumbbell tumors of the cervical spine. Neurosurgery 1996;38(2):294-300

3 Lot G, George B. Cervical neuromas with extradural components: surgical management in a series of 57 patients. Neurosurgery 1997;41(4):813-820, discussion 820-822

4 George B, Lot G. Neurinomas of the first two cervical nerve roots: a series of 42 cases. J Neurosurg 1995;82(6):917-923

5 Hakuba A, Komiyama M, Tsujimoto T, et al. Transuncodiscal approach to dumbbell tumors of the cervical spinal canal. J Neurosurg 1984;61(6):1100-1106

6 Verbiest H. A lateral approach to the cervical spine: technique and indications. J Neurosurg 1968;28(3):191-203

7 Eden K. The dumbbell tumors of the spine. $\mathrm{Br}$ J Surg 1941;28:549-570

8 Asazuma T, Toyama Y, Maruiwa H, Fujimura Y, Hirabayashi K. Surgical strategy for cervical dumbbell tumors based on a three-dimensional classification. Spine 2004;29(1):E10-E14

9 Jiang L, Lv Y, Liu XG, et al. Results of surgical treatment of cervical dumbbell tumors: surgical approach and development of an anatomic classification system. Spine 2009;34(12):1307-1314

10 Dubuisson AS, Kline DG, Weinshel SS. Posterior subscapular approach to the brachial plexus. Report of 102 patients. J Neurosurg 1993;79(3):319-330

11 McCormick PC. Anatomic principles of intradural spinal surgery. Clin Neurosurg 1994;41:204-223

12 Seppälä MT, Haltia MJ, Sankila RJ, Jääskeläinen JE, Heiskanen O. Long-term outcome after removal of spinal neurofibroma. J Neurosurg 1995;82(4):572-577 